\title{
A fresh look at the confinement mechanism
}

\section{Kurt Langfeld*}

School of Computing \& Mathematics, University of Plymouth

Plymouth PL4 8AA, United Kingdom

E-mail: kurt.langfeldeplymouth.ac.uk

Topological configurations, monopoles and vortices, successfully describe quark confinement and the spontaneous breakdown of chiral symmetry. Despite their infinite action, these configurations are relevant due to a subtle cancelation between action and entropy. A natural explanation for this intrinsic fine-tuning is that smooth low action configurations exist which confine and which appear as singular topological objects in certain gauges. To reveal these confining semi-classical configurations, a new cooling method is proposed which largely reduces the action while preserving the asymptotic quark-antiquark potential. First numerical results for a SU(2) gauge theory show that confining configurations with an average plaquette as high as 0.95 do exist.

International Workshop on QCD Green's Functions, Confinement, and Phenomenology - QCD-TNT09 September 07 - 112009

ECT Trento, Italy

\footnotetext{
* Speaker.
} 


\section{Introduction}

Two low energy phenomena of Quantumchromodynamics (QCD) predominantly dictate the properties of matter under normal conditions: quark confinement and the spontaneous breakdown of chiral symmetry. It was already realised in the mid seventies that Yang-Mills theories admit topologically non-trivial configurations with finite action called instantons [1, 2, 3, 4]. It soon became clear that a dilute gas [2] or a liquid [4,5] of instantons may explain the spontaneous breakdown of chiral symmetry. After the advent of computer studies of Yang-Mills theories by means of lattice gauge theories, configurations of low action have been constructed by cooling. It has been reported in that straightforward cooling of lattice configurations annihilates the string tension and therefore quark confinement [6]. Direct evidence of instanton generated confinement has not yet been established.

The wide spread belief is that a different type of configurations which populate the Yang-Mills vacuum is responsible for quark confinement. For example, colour magnetic monopoles generically exist in certain gauges and might generate quark confinement be means of the dual Meissner effect $[7,8]$. Alternatively, a vacuum populated with percolating vortices which carry flux in the centre of the non-Abelian gauge group would equally well explain quark confinement $[9,10]$. Equipped with rapidly increasing computational resources, these ideas were put to the test using lattice gauge simulations. It turned out that the early definitions of the centre vortices failed the quantitative test: the properties of those vortices were strongly dependent on the UV cutoff of the Yang-Mills theory. An important novel definition of the vortex matter was firstly proposed in [11, 12, 13], and the connection of those vortices has been established using lattice gauge simulations. It was pointed out for the first time in $[14,15]$ that these vortices are sensible in the continuum limit when the UV regulator of the theory is removed. In particular for the gauge group SU(2), this vortex texture of the vacuum explains many of the low energy features of Yang-Mills theory. In particular, deconfinement at finite temperatures appears as a vortex depercolation transition $[16,17,18]$, and a close connection between quark confinement and spontaneous chiral symmetry breaking has been found: Removing vortices restores chiral symmetry as realise from the quark condensate [19], from the spectral density of the quark operator [20] or from an inspection of the Landau gauge quark propagator [21]. If the discretised version of the quark operator possesses acceptable chiral properties even for singular vortex background fields, vortex only configurations also show spontaneous chiral symmetry breaking [22]. Up to now, the vortex picture for the gauge group SU(3) is less compelling: Centre projection falls short to reproduce the full string tension ('2/3'-problem) [23], and the tight connection between confinement and spontaneous chiral symmetry breaking seems to be lifted [24]. The '2/3'-problem is absent if the Laplacian Centre gauge is used to define the vortex matter [25]. As pointed out in [26, 23], however, the Laplacian vortex matter is not independent from the UV regulator. The practical consequences are that the vortex removed model theory has lost the property of asymptotic freedom and generically suffers from a low signal to noise ratio. Further SU(3) studies are needed to resolve these issues.

Both, in $\mathrm{SU}(2)$ and $\mathrm{SU}(3)$, the scaling of vortex matter arising from the maximal centre gauge is a remarkable feature of the theory: the infinite action of the vortex world sheets is balanced by an equally large entropy [27, 28]. Since these vortices are defined in a particular gauge, i.e., the so-called maximal centre gauge, a natural explanation of this intrinsic fine tuning would be that 
there is a smooth low action analogue of the vortex configurations which equally well induce confinement. Unraveling these semi-classical configurations would facilitate an analytic first principle understanding of confinement.

In this paper, we report a novel cooling technique which strongly reduces the action of the lattice configurations, but which preserves the long distance behaviour of the static quark antiquark potential and thus quark confinement.

\section{Confinement preserving cooling}

Throughout this paper, we adopt the lattice regularisation of SU(2) Yang-Mills theory. Degrees of freedom are the matrices $U_{\mu}(x)$ associated with the link of the space-time lattice with lattice spacing $a$. We use the Wilson action

$$
S_{\mathrm{wil}}=-\beta \sum_{x, \mu>v} \frac{1}{2} \operatorname{tr}\left[U_{\mu}(x) U_{v}(x+\mu) U_{\mu}^{\dagger}(x+v) U_{v}^{\dagger}(x)\right] .
$$

For our cooling method below, we need to address the static quark-antiquark potential $V(r)$ in terms of un-smeared Wilson loops. The expectation value of a rectangular Wilson loop $W[r, t]$ with extent $r$ in spatial and $t \gg 1 / m_{G}$ in time direction is related to the static potential by:

$$
\langle W[r, t]\rangle=\left|\left\langle 2 \mid \Omega_{2}\right\rangle\right|^{2}(r) \exp \{-V(r) t\},
$$

where $m_{G}$ is the mass gap of the theory, $|2\rangle$ is the state of two heavy, axial-dressed quarks [29, 30, 31] and $\left|\Omega_{2}\right\rangle$ is the true ground state in the quark-antiquark channel. For axial-dressed quarks, the overlap $\left|\left\langle 2 \mid \Omega_{2}\right\rangle\right|^{2}(r)$ is a small number which exponentially decreases with increasing $r$ [30]. It is therefore strongly advisable to use Coulomb dressing or dressing via APE smearing, which both improve on the overlap, if the aim is the calculation of the static potential.

The idea central to our novel cooling is to minimise the action (2.1), but to keep fixed the static potential $V\left(r \geq r_{c}\right)$. For our method, it will turn out below that fixing the potential for $r=r_{c}$ is sufficient to keep $V\left(r \geq r_{c}\right)$ unchanged. Hence, $r_{c}$ is a new scale which comes into play, and which is reminiscent of the so-called cooling radius employed in [32] for elaborate cooling. A straightforward but wrong idea would be to constrain the Wilson loop expectation value $\langle W(r=$ $\left.\left.r_{c}, t\right)\right\rangle$ during cooling. Since the size of the right hand side of (2.2) is dominated by the overlap rather than the exponential, this would merely imply that we freeze the poor overlap. This is not what we want. By contrast, cooling the lattice configuration should increase the overlap of the trial state with the true ground state. To this aim, we consider the ratio

$$
\frac{\langle W[r, t+1]\rangle}{\langle W[r, t]\rangle}=\exp \{-V(r) a\} .
$$

A valid method would be to constrain the above ratio for $r=r_{c}$. Since the numerator and the denominator consist of an average over many lattice configurations, this method is hardly feasible. To circumvent this practical problem, we firstly note that the Wilson loop $W[r, t](x)$ for a given lattice configuration depends on the position $x$ on the lattice. The configuration average, however, 
is position independent due to translation invariance ${ }^{1}$. Consider now two Wilson loops at $x$ and $y$ with a separation much bigger than the inverse mass gap. We then find

$$
\langle W[r, t](x) W[r, t](y)\rangle \approx\langle W[r, t](x)\rangle\langle W[r, t](y)\rangle, \quad|x-y| \gg \frac{1}{m_{G}} .
$$

For pure Yang-Mills theory, the mass gap $m_{G}$ is set by the mass of the lightest $O^{++}$glueball implying that $\xi:=1 / m_{G}$ is short ranged. This implies that Wilson loops separated by distances larger than $\xi$ are statistically independent. For large lattices, i.e., $N a \gg \xi$, we might therefore approximate the average over lattice configurations by an average over space time (and orientation; not shown)

$$
\langle W[r, t](x)\rangle \approx \frac{1}{6 N^{4}} \sum_{x} W[r, t](x) .
$$

The total action which we are going to minimise is given by

$$
S_{\text {all }}=S_{\text {wil }}+\lambda \frac{\sum_{x} W\left[r_{c}, t+1\right](x)}{\sum_{x} W\left[r_{c}, t\right](x)},
$$

where $\lambda$ is a Lagrange multiplicator which ensures that

$$
R:==\frac{\sum_{x} W\left[r_{c}, t+1\right](x)}{\sum_{x} W\left[r_{c}, t\right](x)}=\text { constant }
$$

during cooling. Any sufficiently large value for $t$ should work. We have chosen

$$
t=r_{c}
$$

throughout this paper. The action (2.5) can be minimised by standard means such as steepest decent method. The outline of the cooling approach is as follows:

1 Generate a thermalised lattice configuration $\left\{U_{\mu}\right\}$;

2 Calculate the ratio $R$ in (2.6);

3 Calculate a change $\delta U$ of the link configuration which reduces the total action (2.5);

4 Adjust the Lagrange multiplier $\lambda$ to ensure that $r$ has not changed when you implement the change $\delta U$;

5 Repeat steps 2-5 until the action (2.5) does not change anymore;

6 Start again with step 1 to generate an new cooled configuration.

In order to show that this approach is feasible, we here just report the change of the action $\delta S$ when the particular link $U_{\mu}(x)$ is changed to $\delta U$ while all other links are kept fixed:

$$
\begin{aligned}
\delta S & =\delta U_{\mu}(x)\left[-\beta B_{\mu}(x)+\lambda \frac{C_{\mu}\left[r_{c}, r_{c}+1\right](x)}{\sum_{x} W\left[r_{c}, r_{c}\right](x)}-\lambda \frac{\sum_{x} W\left[r_{c}, r_{c}+1\right](x)}{\left\{\sum_{x} W\left[r_{c}, r_{c}\right](x)\right\}^{2}} C_{\mu}\left[r_{c}, r_{c}\right](x)\right], \\
B_{\mu}(x) & =\sum_{v \neq \mu} U_{v}(x+\mu) U_{\mu}^{\dagger}(x+v) U_{v}^{\dagger}(x) .
\end{aligned}
$$

Thereby, the $B_{\mu}(x)$ are the well known staples, and $C_{\mu}\left[r_{c}, t\right](x)$ is the sum of cut-open Wilson loops $W\left[r_{c}, t\right]$ starting at point $x+\mu$ and ending at point $x$.

\footnotetext{
${ }^{1}$ In practical calculations, we sum over all positions to increase the signal-to-noise ratio.
} 


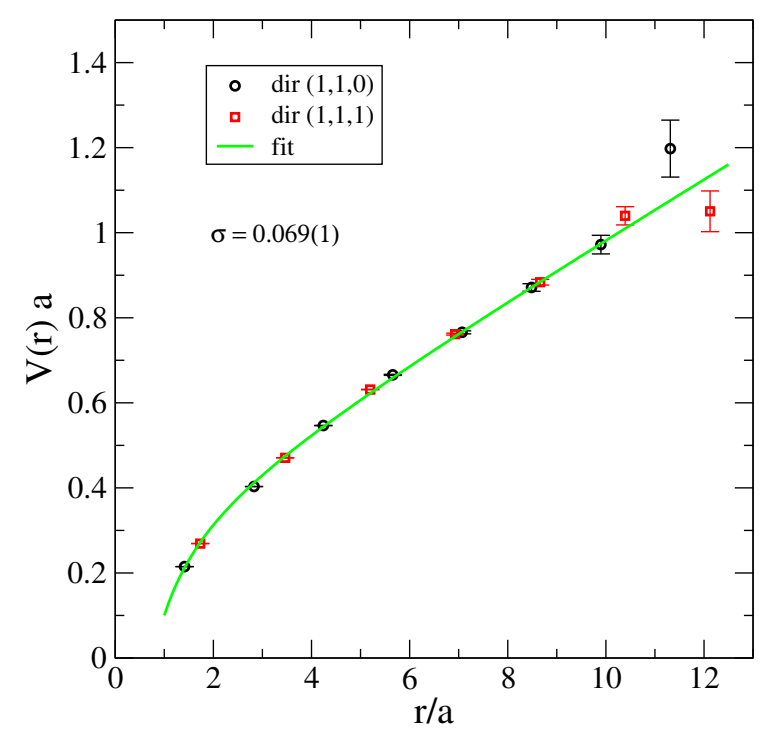

Figure 1: Static quark-antiquark potential obtained from cooled configurations.

\section{Numerical results:}

First numerical results were obtained for a $16^{4}$ lattice for a gauge group $\mathrm{SU}(2)$ with $\beta=2.4$. The cooling radius was fixed to $r_{c}=3$. We find that after 150 cooling steps further changes of the action are tiny.

We firstly checked whether we recover the correct asymptotic behavior of the static quarkantiquark potential from cooled configurations. To this aim, we evaluated the expectation value of rectangular Wilson loops averaged over cooled configuration. The would-be static potential $\bar{V}(r)$ is then obtained from

$$
-\ln \langle W[r, t]\rangle_{\text {cooled }}=\bar{V}(r) t-\operatorname{function}(r), \quad t \gg 1 / m_{G} .
$$

For large values $t>t_{f}$, this function is fitted to a linear function. If the $\chi^{2} /$ dof is not satisfactory, a possible cure would be to increase $t_{f}$. We studied the cases where the quark and the antiquark are aligned along the main cubic axis ((100)-direction) as well as along the diagonals (110) and (111). It turns out that the linear fit was never satisfactory for the orientation (100) of quark and antiquark while this problem did not occur for the diagonal directions. Further studies will be needed to trace out the origin of this effect. For the moment, we keep in mind that the cooled configurations might be plagued by sizable rotational symmetry breaking effects. Because of the constraint during cooling, we ensure that $\bar{V}\left(r_{c}\right)=V\left(r_{c}\right)$. The crucial question is whether $\bar{V}=V$ also holds for $r>r_{c}$. Our numerical result obtained from 640 cooled configurations is shown in figure 1 . The fitted string tension is given by 0.069 (1) which compares quite well with value $0.075(2)$ from full configurations. After a sufficiently large amount of cooling steps ( $>150$ for my setup), the action 


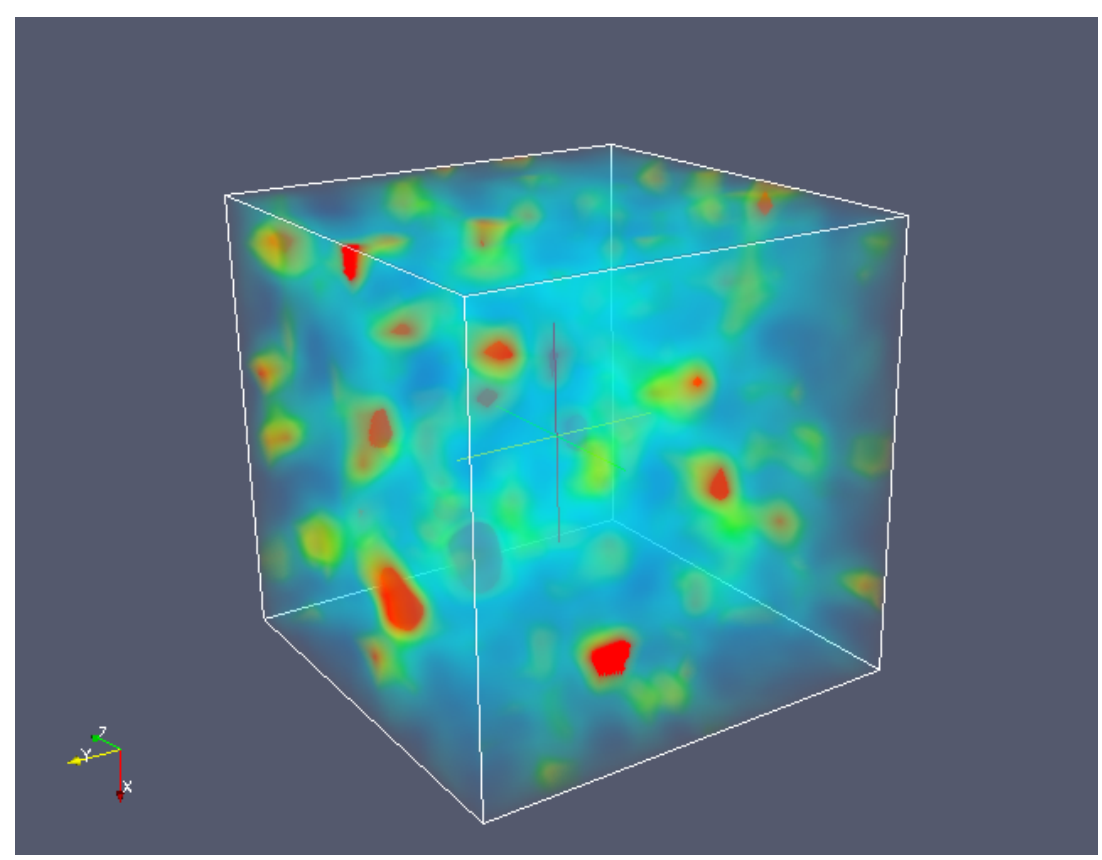

Figure 2: Action density obtained from cooled configurations with cooling radius $r_{c}=3 a, \beta=2.4,20^{4}$ lattice.

density seems to stabilise. The constraint during cooling prevents the configurations to become the perturbative vacuum with trivial plaquettes only. An example of such a cooled configuration (cooling radius $r_{c}=3 a, \beta=2.4$ ) is shown in figure 2 . We clearly observe a lump type structure in the vacuum. The overall achieved average plaquette of this configuration has been $\approx 0.95$.

\section{Conclusions}

Over the last two decades, topological configurations, such as monopoles and vortices, have been successfully linked to the confinement phenomenon in Yang-Mills theories. It has been observed in the recent past that those degrees of freedom also describe the spontaneous breakdown of chiral symmetry. These configurations are relevant due to a subtle cancelation between action and entropy. A natural explanation for this intrinsic fine tuning is that this singular configurations have a low action counter part in a different gauge. In order to unravel these semi-classical configurations, a new type of cooling was introduced which largely reduces the action but preserves the asymptotic behavior of the quark potential. The viability of the new cooling method has been explored for the $\mathrm{SU}(2)$ gauge theory for lattices as big as $20^{4}$. A lump type vacuum structure has been observed using the action density.

Future studies will address the questions: Does the action density of the cooled configurations scale like $a^{4}$ ( $a$ the lattice spacing) implying a finite action density in the continuum limit? What is the topological charge of the observed lumps, and what is their relation to instantons and calorons [33]? Does the observed structure match with monopoles and vortices in the corresponding gauges? 


\section{References}

[1] A. A. Belavin, A. M. Polyakov, A. S. Shvarts and Yu. S. Tyupkin, Phys. Lett. B 59 (1975) 85.

[2] C. G. . Callan, R. F. Dashen and D. J. Gross, Phys. Rev. D 17 (1978) 2717.

[3] G. 't Hooft, Phys. Rev. D 14 (1976) 3432 [Erratum-ibid. D 18 (1978) 2199].

[4] E. V. Shuryak, Nucl. Phys. B 203 (1982) 93.

[5] D. Diakonov and V. Y. Petrov, Nucl. Phys. B 245 (1984) 259.

[6] M. I. Polikarpov and A. I. Veselov, Nucl. Phys. B 297 (1988) 34.

[7] G. ’t Hooft, Nucl. Phys. B 190 (1981) 455.

[8] A. S. Kronfeld, M. L. Laursen, G. Schierholz and U. J. Wiese, Phys. Lett. B 198 (1987) 516.

[9] J. M. Cornwall, Nucl. Phys. B 157 (1979) 392.

[10] G. Mack, Phys. Rev. Lett. 45, 1378 (1980).

[11] L. Del Debbio, M. Faber, J. Greensite and S. Olejnik, Nucl. Phys. Proc. Suppl. 53 (1997) 141 [arXiv:hep-lat/9607053].

[12] L. Del Debbio, M. Faber, J. Greensite and S. Olejnik, Phys. Rev. D 55, 2298 (1997) [arXiv:hep-lat/9610005].

[13] L. Del Debbio, M. Faber, J. Giedt, J. Greensite and S. Olejnik, Phys. Rev. D 58 (1998) 094501 [arXiv:hep-lat/9801027].

[14] K. Langfeld, H. Reinhardt and O. Tennert, Phys. Lett. B 419 (1998) 317 [arXiv:hep-lat/9710068].

[15] M. Engelhardt, K. Langfeld, H. Reinhardt and O. Tennert, Phys. Lett. B 431 (1998) 141 [arXiv:hep-lat/9801030].

[16] K. Langfeld, O. Tennert, M. Engelhardt and H. Reinhardt, Phys. Lett. B 452 (1999) 301 [arXiv:hep-lat/9805002].

[17] M. Engelhardt, K. Langfeld, H. Reinhardt and O. Tennert, Phys. Rev. D 61 (2000) 054504 [arXiv:hep-lat/9904004].

[18] K. Langfeld, Phys. Rev. D 67 (2003) 111501 [arXiv:hep-lat/0304012].

[19] P. de Forcrand and M. D’Elia, Phys. Rev. Lett. 82 (1999) 4582 [arXiv:hep-lat/9901020].

[20] J. Gattnar, C. Gattringer, K. Langfeld, H. Reinhardt, A. Schafer, S. Solbrig and T. Tok, Nucl. Phys. B 716 (2005) 105 [arXiv:hep-lat/0412032].

[21] P. O. Bowman, K. Langfeld, D. B. Leinweber, A. O’ Cais, A. Sternbeck, L. von Smekal and A. G. Williams, Phys. Rev. D 78 (2008) 054509 [arXiv:0806.4219 [hep-lat]].

[22] R. Hollwieser, M. Faber, J. Greensite, U. M. Heller and S. Olejnik, Phys. Rev. D 78 (2008) 054508 [arXiv:0805.1846 [hep-lat]].

[23] K. Langfeld, Phys. Rev. D 69 (2004) 014503 [arXiv:hep-lat/0307030].

[24] D. B. Leinweber, P. O. Bowman, U. M. Heller, D. J. Kusterer, K. Langfeld and A. G. Williams, Nucl. Phys. Proc. Suppl. 161 (2006) 130.

[25] P. de Forcrand and M. Pepe, Nucl. Phys. B 598 (2001) 557 [arXiv:hep-lat/0008016].

[26] K. Langfeld, H. Reinhardt and A. Schafke, Phys. Lett. B 504 (2001) 338 [arXiv:hep-lat/0101010]. 
[27] F. V. Gubarev, A. V. Kovalenko, M. I. Polikarpov, S. N. Syritsyn and V. I. Zakharov, Phys. Lett. B 574 (2003) 136 [arXiv:hep-lat/0212003].

[28] V. I. Zakharov, arXiv:hep-ph/0306262.

[29] T. Heinzl, A. Ilderton, K. Langfeld, M. Lavelle, W. Lutz and D. McMullan, Phys. Rev. D 77 (2008) 054501 [arXiv:0709.3486 [hep-lat]].

[30] T. Heinzl, A. Ilderton, K. Langfeld, M. Lavelle, W. Lutz and D. McMullan, Phys. Rev. D 78 (2008) 034504 [arXiv:0806.1187 [hep-lat]].

[31] T. Heinzl, A. Ilderton, K. Langfeld, M. Lavelle and D. McMullan, Phys. Rev. D 78 (2008) 074511 [arXiv:0807.4698 [hep-lat]].

[32] P. de Forcrand, M. Garcia Perez and I. O. Stamatescu, Nucl. Phys. B 499 (1997) 409 [arXiv:hep-lat/9701012].

[33] T. C. Kraan and P. van Baal, Phys. Lett. B 435 (1998) 389 [arXiv:hep-th/9806034]. 